\title{
Vibration decrease of electromechanical actuators based on roller screw mechanisms
}

\author{
Valentin Morozov ${ }^{1}$, Pavel Fadeev ${ }^{1}$, Dmitri Shtych ${ }^{1}$, Leonid Belyaev ${ }^{1}$, and Alexey Zhdanov ${ }^{1, *}$ \\ ${ }^{1}$ Department of Mechanical Engineering, Vladimir State University named after Alexander and Nikolay Stoletovs, Vladimir, Russia
}

\begin{abstract}
The reduction of the vibratory activity of the roller screw mechanism (RSM) for converting a rotational movement into a translational movement is discussed. A mathematical model of vibratory activity roller screw mechanisms on the basis of elastic compliances was developed. The resulting equation for calculating the natural frequencies of the basic RSM elements - screw, rollers and nut was obtained. It is proved that the RSM has a low vibroactivity that allows you to create mechatronic actuators, the maximum level of vibration does not exceed 60 to $62 \mathrm{~dB}$, which is 11-16 dB below the level of vibratory activity gear mechanisms
\end{abstract}

\section{Intruduction}

Recently, planetary roller-screw mechanisms (RSM) have become widely used among executive mechanisms used for converting rotary movement into linear displacement [1-6]. In papers [2, 3,6] we have shown methods for calculating the main technical characteristics of those mechanisms. At the same time, an important actuators characteristic, often determining the applicability of a particular mechanism, is its vibroactivity and, as a result, the noise generated. The screw gear smoothness is the cause of decreased RSM vibroactivity and noise and makes those mechanisms promising for use. As for RSM, the closest analogues for the design models are planetary gear mechanisms $[4,5]$ and rolling bearings $[6,7]$ which vibrational processes have been studied sufficiently. However, the use of calculation procedures developed here and practical recommendations for the RSM vibrations reduction is unjustified, because of the changing characteristics and difference between screw mechanisms [8,9]. Let us consider the mechanism option where the screw-rollers support interface is made with multistart threads and has end-capping geared rims. The mechanism structure (Fig.1) comprises screw 1 installed in bearings fixed in housing 2. On the screw and rollers geared rims 6 were cut. The screw is mating the thread rolls 3 through the thread and through the gear rims 6 , and the rollers interact with the nut 4 thread. The casing is fixed to the base 5 . The problems solved during RSM vibroactivity study are as follows: determining vibration sources, their frequencies and amplitudes, calculating the forced vibrations spectrum, determining the audio range vibration levels accounted for by the RSM casing.

\section{Materials and methods}

Out of the many oscillation theory methods, the most suitable method for solving the tasks in hand is the dynamic compliance method. The method is as follows. The mechanism is divided into a number of simple subsystems. As for the RSM the following subsystems are distinguished: screw, rollers, nuts and housing (Fig. 2).

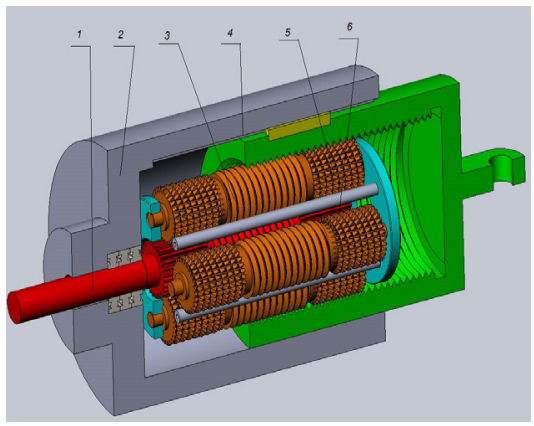

Fig. 1. Structure of Roller Screw Mechanism

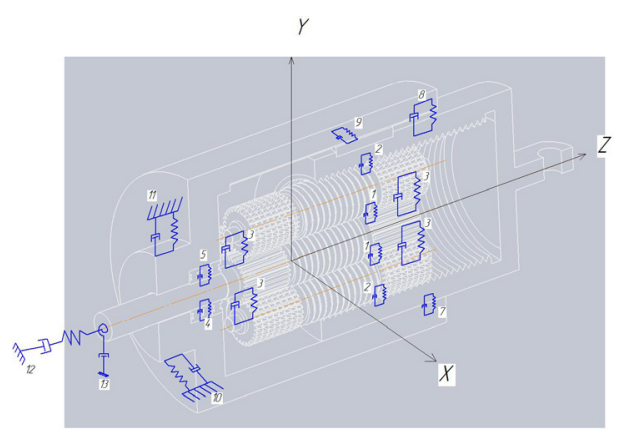

Fig. 2. Diagram of RSM; elastic connection:

1 - screws and clips; 2 - roller and nuts; 3 - screw and toothed roller in the circuit; 4 - screw-on body $X$ axis; 5 - the screw-on housing axis $\mathrm{Y} ; 6$ - the screw-on housing axis $\mathrm{Z} ; 7$ - a nut body on X-axis; 8 - a nut body on Y-axis; 9 - key - keyway; 10 body-reliance on the $\mathrm{X}$ axis; 11 - body-reliance on the $\mathrm{Y}$ axis; 12 - body-reliance on the $\mathrm{Z}$ axis; 13 - body-support in angle $\varphi$.

\footnotetext{
*Corresponding author: zhdanov@,vlsu.ru
} 
At the points of mechanism dismemberment into simple subsystems constraining forces are applied that vary with some degree of frequency, forced vibrations resulting from the action of those harmonic constraining forces are calculated separately for each subsystem and displacements of points along which the linkages are broken in the linkages of those bonds are located. After that, the strain compatibility equations were set up for each elastic linkage (the number of equations was equal to the number of broken linkages), the frequency equation was solved and displacements value in selected joint coordinates were determined.

\section{Vibroactivity calculation of RSM}

During RSM operation, the most important sources of vibration are threaded surfaces manufacturing errors. These are zero lead-ins $(n=0)$ threaded elements eccentricities, ovalities, facetings and undulations, and in case of threaded elements with $\mathrm{n}>0$ to these must be added periodic pitch errors [2]. The whole set of errors leads to periodic threaded surface generator position motions which we shall hereafter refer to as undulation.

We will make the following assumptions: the deformation in the RSM threaded elements contact follows the Hertz-Belyaev law; the load-side axial load is constant and uniformly distributed over all the contact points; the amount of threaded elements working surfaces undulation is within the elastic deformation limits. We will establishing the exciting force values dependence on the undulation amount.

The normal force at each threaded surfaces contact point $\mathrm{P}_{\mathrm{H}}$ causes elastic deformation $\delta$.

The threaded surfaces undulation can be expanded into Fourier series with each i-th undulation harmonic causing additional elastic deformation $\delta_{\mathrm{zi}}$.

$\delta=\delta_{1}+\sum \delta_{z i}$ The dependence of elastic deformation on the normal force (in case of linear contact of two bodies) is nonlinear [4]. In order to use in a linear equation system additional force caused by the undulation and vibrating the mechanism we will find the linear approximation by way of using direct linearization method.

$$
\delta=c \cdot q
$$

Where $c=K_{1}\left(K_{2}-\ln q_{\max }+\frac{1}{3}\right) ; K_{1}=0,579 \mathrm{E}$; $K_{2}=\frac{\ln \left(4 E\left(r_{1}+r_{2}\right)\right)}{1,522^{2} \sin \alpha}+0,814 ; q_{\max }-$ maximum specific

load; $E$ - reduced modulus of elasticity; $r_{1}$ and $r_{2}$ - the radii of curvature of the contacting surfaces; $\alpha$ - half thread angle.

Given that, where $L_{\mathrm{KOH}}$ - the length of the contact line, the variable load is in contact, is the driving force of vibration $\left(P_{H}\right)$ ), it is equal to:

$$
P_{H_{i}}=\frac{\left(a_{i} \cos i w t+b_{i} \sin i w t\right) L_{K O H}}{c}
$$

Let us introduce two coordinate systems: one fixed XOY coordinate system and a $\mathrm{X}^{\prime} \mathrm{OY}^{\prime}$ system rotating relative to the first one with the roller radius vector rotary speed, i.e., connected to the separator, which rotary speed is calculated using the following formula [2]:

$$
\omega_{P}^{o}=\frac{\omega_{B}(1+u)}{2}
$$

where $\omega_{B}$ - angular screw speed; $u=d_{p} / d_{B}, d_{p}, d_{B}$ roller and screw diameters respectively.May the screw rotate at a rotary speed $\omega_{\mathrm{B}}$ and have undulation of i-th harmonic. We will consider the mechanism in the XOY coordinate system.

The angle between the $\mathrm{j}$-th roller contact point and the screw in the moving coordinates is

$$
B_{i}=\omega_{\text {OTH }} t+2 \pi \frac{(j-1)}{n}
$$

where $\omega_{\text {Отн - rotor angular velocity in the moving }}$ coordinate system (speed of rotation of the screw with respect to the separator) $\omega_{\text {OTH }}=-\left(\omega_{B}-\omega_{P}^{0}\right)$. The normal force arising from the $\mathrm{i}$-th screw harmonic undulation on all cutting threads $(1 \leq \mathrm{K} \leq \mathrm{m})$

$$
F_{n i j}=\frac{l_{K O H}}{l}\left(\sum_{K=1}^{m} \alpha_{i K}^{b} \cos i \varphi_{B_{j}}+\sum_{K=1}^{m} b_{i K}^{b} \sin i \varphi_{B j}\right) .
$$

Using (1) and turning to a fixed coordinate system we will define after the transformation the joint force along the $\mathrm{Z}$ axis, which causes axial vibrations.

$$
F_{z i}=\frac{l_{\text {KOH }} n \cos \alpha}{c}\left(\sum_{K=1}^{m} \alpha_{i K}^{b} \cos i \omega_{\text {OTH }} t+\sum_{K=1}^{m} b_{i K}^{b} \sin \omega_{\text {OTH }} t\right)(2)
$$

where $i=N \cdot n,(N=1,2,3, \ldots)$. By finding the normal force projection at the $\mathrm{j}$-th roller and the screw contact point on the $\mathrm{Y}$ axis, let's find the forces causing vibrations in radial axis. Similarly, we will determine the constraining forces from the rollers and the nuts threaded surfaces undulation that causes vibrations in radial and axial directions. Frequencies of the constraining forces generated by the threaded elements undulation are used to calculate the RSM vibroactivity/.

Let's separate RSM screw from the elastic system (Fig. 3). It is necessary to determine the dynamic compliance coefficients at the points where it joins the conjugate elements through elastic bonds (Figure 3). To solve this problem, it is necessary to create the selected element (screw) equations of motion under the impact of harmonic forces applied at the bonds cut-off points. In this case, regarding the screw, these forces will act in the normal direction at the screw points of contact with the $n$ rollers on the thread surface, at the screw points of contact with the $n$ rollers in the gear coupling and in the direction of the bearing assembly major rigidity, since, 
generally speaking, the bearing assembly design is unknown.

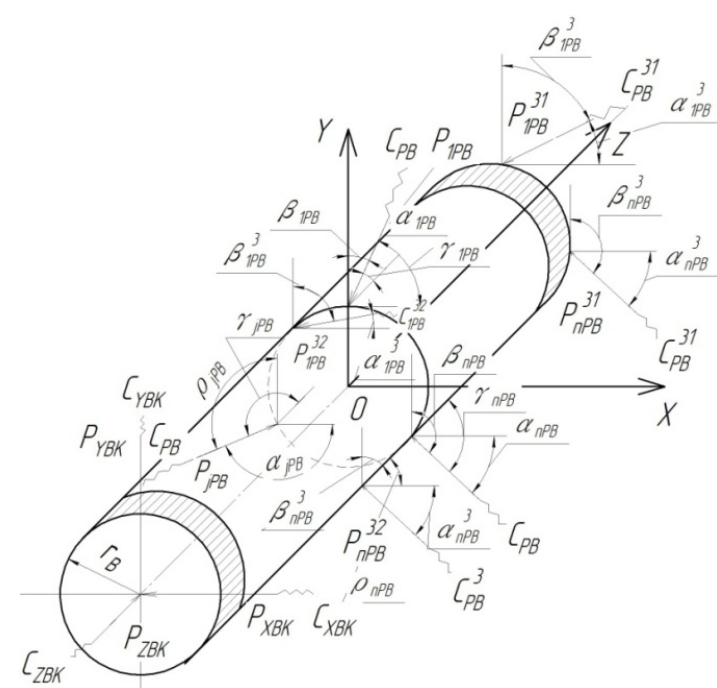

Fig. 3. Scheme of RSM screw

The equations of motion in a plane perpendicular to the screw axis and in the axial direction have the form:

$$
\begin{aligned}
& m_{B} \cdot \ddot{Y}+b_{y} \cdot \dot{X}= \\
& {\left[\sum_{j=1}^{n} P_{j P B} \alpha_{j P B}+\sum_{j=1}^{n}\left(P_{j P B}^{31}+P_{j P B}^{32}\right) \alpha_{j P B}^{3}+P_{X B K} \cdot 1\right] \cos \omega t} \\
& m_{B} \cdot \ddot{Y}+b_{y} \cdot \dot{Y}= \\
& {\left[\sum_{j=1}^{n} P_{j P B} \beta_{j P B}+\sum_{j=1}^{n}\left(P_{j P B}^{31}+P_{j P B}^{32}\right) \beta_{j P B}^{3}+P_{Y B K} \cdot 1\right] \cos \omega t} \\
& m_{B} \cdot \ddot{Y}+b_{y} \cdot \dot{Z}=\left[\sum_{j=1}^{n} P_{j P B} \gamma_{j P B}+P_{Z B K} \cdot 1\right] \cos \omega t \\
& j_{B} \cdot \ddot{\varphi}+b_{\varphi} \cdot \dot{\varphi}=\left[\sum_{j=1}^{n} P_{j P B} \varphi_{j P B}+\sum_{j=1}^{n}\left(P_{j P B}^{31}+P_{j P B}^{32}\right) \varphi_{j P B}^{3}\right] \cos \omega
\end{aligned}
$$

where $m_{B}$ - weight screws; $j_{B}$ - moment of inertia about the axis of the screw; $P_{j P B}$ harmonic amplitudes of the forces acting on the screw at points of contact with the rollers; $P_{j P B}^{31} ; P_{j P B}^{32}$ the amplitude of the harmonic forces acting on the screw at the points of engagement with the toothed rollers; $b_{x}, b_{y}, b_{z}, b_{\varphi}$ damping coefficients that take into account the power dissipation in the direction of oscillation of the generalized coordinates; $P_{X B K}, P_{Y B K}, P_{Z B K}$ amplitude harmonic force in the contact-screw housing; $\alpha_{j P B}, \beta_{j P B}, \gamma_{j P B}$, $\boldsymbol{\varphi}_{j P B}, \alpha_{j P B}^{3}, \beta_{j P B}^{3}, \varphi_{j P B}^{3}$ points and the direction cosines of the unit vectors directed along the axes $x j$ springs; $\omega$ the frequency of the driving force; $X, Y, Z, \varphi$ coordinates of the center of gravity and rotation motion with respect to the screw axis $O Z$.

In the motion equation system (3) the amplitudes of the harmonic forces affecting the screw at the points of gear coupling with the rollers $P_{j P B}^{31} ; P_{j P B}^{32}$ are directed along the coupling line at any given time $t$. The equations of screw motion were set up in the XOY coordinate system rotating with the angular velocity $\omega_{p}^{0}$. The transition to the fixed XOY coordinate system will take place when determining the vibratory acceleration amplitudes. The same calculation procedure was also applied when considering other RSM subsystems motion. The spot solution of equation (3) will be in the form of:

$$
X=A \cos \omega t \sin \omega t
$$

In matrix form the equilibrium equations system has the form of:

$$
M_{B} X_{B}=R_{B} P_{B}
$$

where $M_{B}$ mass matrix of the screw; $X_{B}$ vector amplitudes screw displacement of the center of gravity; $P_{B}$ vector amplitudes forced harmonic forces; $R$ matrix of direction cosines. The movements of J-th points along which the bonds were broken in the direction of those links (in the force line direction

$$
U_{B}=R_{B}^{T} X_{B}
$$

where $U_{B}$ - motion vector points j-points; transposed matrix .Solving equation (5) in terms of $X_{B}$ and multiplying both its parts by a matrix $M_{B}^{-1}$ inverse to the matrix we obtain:

$$
X_{B}=M_{B}^{-1} R_{B} P_{B}
$$

Let plug (7) into the equation (6) and we obtain a link between the $\mathrm{j}$-th points movements and the forces acting in those directions

$$
U_{B}=R_{B}^{T} M_{B}^{-1} R_{B} P_{B}
$$

when $E_{B}=R_{B}^{T} M_{B}^{-1} R_{B}$ - the coefficient matrix of the dynamic compliance of the screw.Similarly rollers, nuts and casing dynamic compliance coefficients are determined that are used to work out the system for canonical equations subject to no-movement condition at the points where the whole system is divided into subsystems in the direction of those bonds. In matrix form we will obtain the following equation system:

$$
A P=0
$$

when $P$ - column matrix of harmonic forces (reaction of links); A - matrix of dynamic compliance RBM system, where each element is the sum of compliances 
subsystems at the removal of links between them. To find the natural system frequencies of vibration the frequency equation is solved:

$$
\operatorname{det} A=0
$$

When calculating forced vibrations the dynamic compliance method is also used. The general matrix equation for calculating the forced system vibrations has the form of:

$$
A P=A^{*} F
$$

where $A^{*}$ - matrix dynamic compliance system; $P$ vector reactions in relationships; $F$ - column matrix driving forces acting on relations.

$$
F=\left\{F_{1 P B} \ldots F_{n P B} F_{1 P B}^{31} F_{1 P B}^{32} \ldots F_{n \mathrm{Pr}} F_{1 P B}^{31} F_{1 P B}^{32} \ldots \ldots F_{n \mathrm{Pr}}\right.
$$

When calculating the forced system vibrations, the superposition principle is used due to the linearity of equation (11), i.e., the impact of the system of constraining forces of the like harmonics (frequency) were considered separately, which is fully in line with the spectral analysis' objectives. The obtained RSM element of interest vibratory displacement amplitudes were summed.We would also like to note that when calculating the free vibrations, RSM frequencies that make part of the matrix $A$ are the natural vibration frequencies and are determined from equation (9) and in case of forced vibrations those frequencies are preset (known) frequencies of constraining forces. The general course of forced vibrations calculation by way of dynamic compliance prediction is as follows. In matrix $F$ constraining forces of the same frequency $\omega_{1}$ were taken into account. This frequency value is plugged into dynamical compliance coefficients expressions in matrices $A$ and $A^{*}$. From (11) unknown reactions in the bonds caused by the impact of constraining forces are determined. After that each subsystem can be considered independently of each other. From the equilibrium equations of the interesting subsystem, the amplitude of vibratory displacement in the direction of the required generalized coordinate is determined. Then, if necessary, vibration speed, vibration acceleration and vibration log level can be found later. When changing the constraining force frequency to other one, it is necessary to carry out complete recalculation again, because the dynamic compliance coefficients values in matrices $A$ and $A^{*}$ also change.

As already indicated, the most interesting from the practical point of view are vibrations accounted for by the RSM casing and generating its vibroactivity and noise, as well as vibrations overlapping the output motion and determining the drive operation smoothness.

When analyzing the executive units vibration, it is more convenient to use a third-octave spectrum rather than a narrowband one, because in this case the spectrum record is of more compact form and presents a general picture of the vibration process over a wide frequency range. In this case, the volume of vibration amplitudefrequency spectra calculations was significantly reduced.

The methods presented are convenient for the RSM vibrational activity checking calculations when the exciting forces spectrum can be experimentally obtained. For this purpose, the threaded elements undulation is measured. For example, the undulation of zero lead-in elements can be measured using a profilometer.

This results in obtaining an undulation profilogram of all the cutting threads of the nut, screw and rollers being machined. The obtained data arrays are expanded into Fourier series and the exciting forces amplitudefrequency spectrum is formed. This spectrum was used in the technique under discussion, and the vibration amplitude-frequency spectrum of the particular executive unit, which elements undulation has been measured, was determined.

To develop a methodology for vibroactivity forecasting at the design stage when only tolerances for the production of the main members of a mechanism are set, it is necessary to take into account the likely nature of exciting forces.

\section{Experimental study of RSM vibroactivity}

The item under test was a roller-screw mechanism which structure is shown in Fig. 1. Vibration levels were measured in the frequency range from 20 to $20,000 \mathrm{~Hz}$ using a specially manufactured stand shown in Fig. 4. Sample RSM 2 is fixed on the bracket on the foundation 1. The RSM output rod by a connecting rod 3 is connected to a spring-actuated loading device 4 designed to create forces on the rod of the mechanism. In the loading device a $5.7 \mathrm{kgf} / \mathrm{cm}$ force spring with a $100 \mathrm{~N}$ preloading was used. In the extreme mechanism rod positions the loading device generated forces of up to $350 \mathrm{~N}$

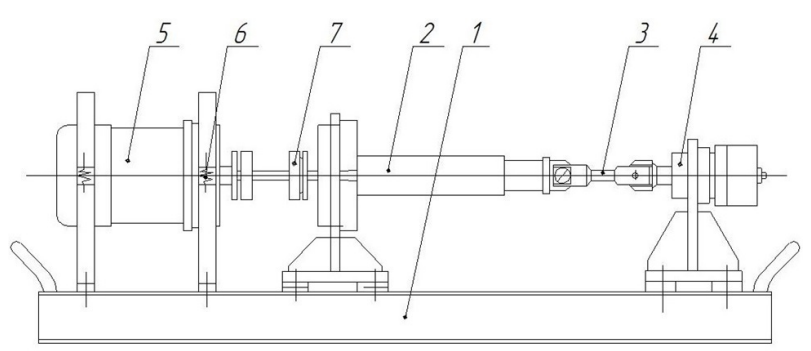

Fig. 4. Test stand for investigation of RSM

Vibration sensors were mounted on the stand basement and on the RSM housing. The vibration parameters were measured in an orthogonal coordinate system in the $\mathrm{OY}$ and $\mathrm{OZ}$ axes direction. Following vibration characteristics were determined during testing: the RSM vibration level in the radial direction (OYdirection); RSM vibration level in the axial direction $\left(\mathrm{Z}^{0}\right.$ direction)Equipment manufactured by Bruel \& Kjaer Company was used for testing. Sensors installation, calibration, preparation for testing, measurements and analysis of results were performed in accordance with the above equipment operating instructions. 
Values of vibration levels in the radial direction are somewhat lower than in Y-direction and are more evenly distributed over frequency bands with no pronounced peak values. At frequencies lower than $80 \mathrm{~Hz}$ vibration levels were not higher than $40 \mathrm{~dB}$, within the range of 250 to $4 \mathrm{kHz}$ their values amounted to 48 to $58 \mathrm{~dB}$. Maximum vibration levels amounted to $65 \mathrm{~dB}$ in frequency bands of 6.3 to $12.5 \mathrm{kHz}$. In case of screw rotation speed increase from 21 to $25 \mathrm{~Hz}$ regarding RSM 1 , the vibration level in the radial direction increases by 2 to $3 \mathrm{~dB}$. Narrowband RSM vibration analysis spectra within the frequency range of 0 to $2000 \mathrm{~Hz}$ shown in Fig. 5 have a high density of vibration frequencies discrete components, which is consequential of a significant number of constraining force harmonics caused by the threaded elements undulation.

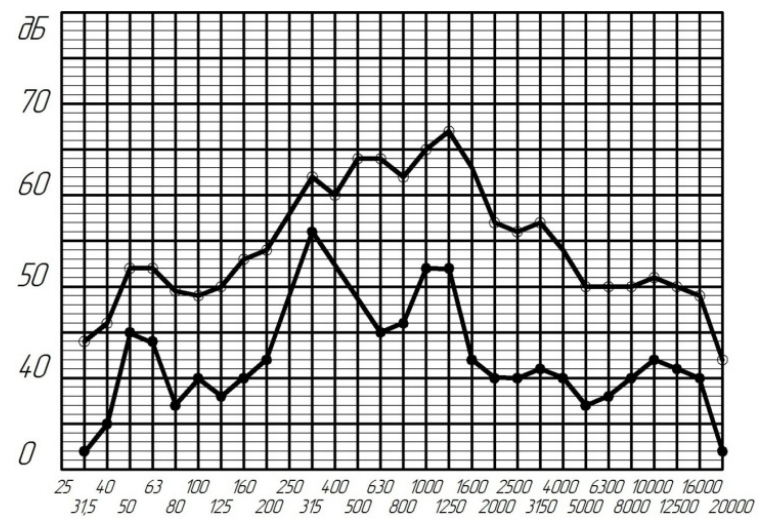

a)

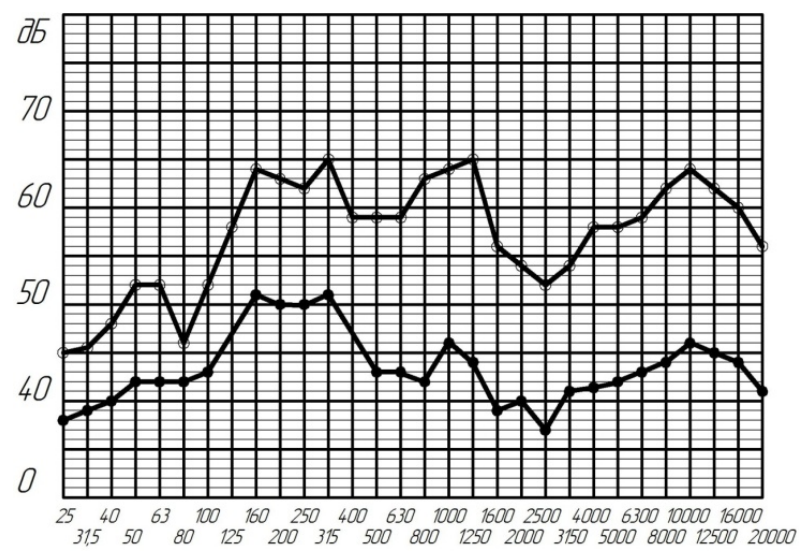

b)

Fig. 5. Third octave spectra RSM vibroactivity: a) axially vibroactivity: b) radial vibroactivity: - no load, $\bigcirc-$ load $380 \mathrm{~N}$

\section{Results and discussion}

Third-octave spectra of vibration levels in the radial and axial directions during RSM 1 and RSM 2 testing were obtained in all modes of interest. Third-octave spectra of vibration levels in the radial direction of the RSM testing at regime are shown as an example in Fig. 6. Analysis of the available spectrograms showed that the values of vibration levels in the radial direction were from 36 to 64 $\mathrm{dB}$ in different third-octave frequency bands. Vibration level maximums are in the frequency ranges of 250 to $315 \mathrm{~Hz}, 800$ to $1000 \mathrm{~Hz}$ and of 8 to $10 \mathrm{kHz}$. The RSM 1 vibration levels in the axial direction have maximum values in the frequency bands range of 63 to100 Hz, 250 to $400 \mathrm{~Hz}, 1$ to $16 \mathrm{~Hz}$ and amount to 53 to 60,68 to 62 and 58 to $62 \mathrm{~dB}$.Studies allowed us to draw following conclusions:

1. RSM have low vibroactivity which allows developing mechatronic drives based on them with the maximum vibration level not exceeding 60 to $62 \mathrm{~dB}$, which is 11 to $16 \mathrm{~dB}$ below the gear mechanisms vibroactivity level.

2. The method developed for calculating RSM vibroactivity allows vibration level predicting at the drives design stage.

3. The study of developed models allows specifying requirements for the accuracy of screw, rollers and nut thread surfaces manufacture. Thus, the vibroactivity of an RSM with the Tolerance Class 1 elements is by $16 \%$ less than those with elements of Tolerance Class 2.

4. The given experimental data showed that the maximum RSM vibration level does not exceed 60 to 62 $\mathrm{dB}$. With the increase of axial load from 0 to $760 \mathrm{No}$, vibration in the radial direction decreases by 2 to $6 \mathrm{~dB}$. When screw rotation speed increases from 21 to $26 \mathrm{~Hz}$, the vibration level increases by 2 to $3 \mathrm{~dB}$.

5. Narrowband vibration analysis showed that the vibration spectrum is continuous with a large number of vibration frequencies discrete components which is due to a significant number of constraining forces harmonics. 6. A comparison of the calculated and experimental vibration levels third-octave spectra showed the adequacy of developed models to the real RSM processes taking place. The difference between calculated and experimental results did not exceed 4 to $10 \mathrm{~dB}$ which is 6 to $16 \%$

\section{References}

1. M. Shangjun, L. Geng, T. Ruiting, Z. Xiaocai, A Mathematical Problems in Engineering (2012)

2. V. Morozov Roller screw mechanisms. Kinematic Characteristics (VISU, Vladimir, 2005)

3. V. Morozov, A. Zhdanov, Roller screw mechanisms. Reliability and durability (Pero, Moscow, 2015)

4. A. Zhdanov, V. Morozov, Procedia Engineering, 150 (2016)

5. V. Morozov, A. Zhdanov, Russian Aeronautics, 59, 1 (2016)

6. V. Morozov, A. Zhdanov, A. Kosterin, Russian Engineering research, 36, 9 (2016)

7. V.V. Kozyrev, Comparison of ball and roller screwnut transmissions, Bulletin of Mechanical Engineering., 11, pp. 31-35 (1983)

8. V.V. Kozyirev, Design theory and methods of design and study of planetary gear-nut threaded rollers and mechatronic units based on them: monograph (Vladimir State University, Vladimir, 2011)

9. D.S. Blinov, M.I. Morozov Promising designs of planetary roller screw mechanisms, Izvestiya vuzov, Mishinostroenie, 3, pp. 62-72 (2013) 